\title{
Diversidad de mamíferos terrestres de talla grande y media de una selva subcaducifolia del noreste de Oaxaca, México
}

\section{Diversity of large and medium sized land mammals of a subcaducifolious tropical forest of north eastern of Oaxaca, Mexico}

\author{
Gabriela Pérez-Irineo ${ }^{\varpi}$ y Antonio Santos-Moreno \\ Laboratorio de Ecología Animal, Centro Interdisciplinario de Investigación para el Desarrollo Integral Regional, Unidad Oaxaca, Instituto Politécnico \\ Nacional. Hornos 1003, 71230 Santa Cruz Xoxocotlán, Oaxaca, México. \\ \gabyirineo@yahoo.com.mx
}

\begin{abstract}
Resumen. El estado de Oaxaca en el sureste de México alberga una biodiversidad extraordinaria. Sin embargo, las actividades humanas han propiciado alteraciones considerables, incluida la disminución de diferentes ambientes naturales, especialmente de los bosques tropicales; por ello es importante documentar la diversidad de estos ambientes, así como su respuesta a las transformaciones antropocéntricas. Este trabajo tiene como objetivo estimar la diversidad de mamíferos terrestres en una selva mediana en el noreste del estado de Oaxaca, México. Se recorrieron 3 senderos para la búsqueda de rastros, captura de organismos, avistamientos y fototrampeo de septiembre de 2007 a agosto de 2008. Se registraron 15 especies de 6 órdenes, 11 familias y 14 géneros, entre ellas 7 nuevas para la región. Las especies más abundantes fueron Nasua narica, Pecari tajacu, Dasyprocta mexicana, Cuniculus paca y Leopardus pardalis. A pesar de presentar un índice de diversidad bajo con respecto a otros estudios comparables, la presencia de especies consideradas en riesgo por la legislación mexicana, como L. pardalis, L. wiedii, Eira barbara y Tamandua mexicana hacen a la zona valiosa para la conservación de la diversidad en la región.
\end{abstract}

Palabras clave: abundancia, riqueza de especies, Tuxtepec.

\begin{abstract}
The state of Oaxaca, in southeast Mexico is home to an extraordinary biodiversity. But human activities have led to significant changes, including reduction of different natural habitats, especially tropical forests, so it is important to document the diversity of these environments, and their response to the anthropocentric changes. Therefore, this study aimed to estimate the diversity of terrestrial mammals in a tropical forest in northeastern of the state of Oaxaca, Mexico. Three transects were walked to search for tracks, catches of organisms, sightings, and phototrapping from September 2007 to August 2008. There were recorded 15 species of 6 orders, 11 families, and 14 genera, 7 of this not previously known in the region. The most abundant species were Nasua narica, Pecari tajacu, Dasyprocta mexicana, Cuniculus paca, and Leopardus pardalis. Despite the low value of the diversity index compared with similar studies, the presence of species considered at risk by the Mexican law as L. pardalis, L. wiedii, Eira barbara, and Tamandua mexicana make the area valuable for the conservation of the diversity of the region.
\end{abstract}

Key words: abundance, species richness, Tuxtepec.

\section{Introducción}

El estado de Oaxaca en México es reconocido por la gran biodiversidad que presenta, y que se debe, entre otros factores, a una alta heterogeneidad ambiental y topográfica, y a la amplia variedad de tipos de vegetación (Flores y Manzanero, 1999). Sin embargo, la perturbación antropogénica ha provocado la transformación y disminución de ambientes originales. Tal es el caso de los bosques tropicales en el estado (Challenger, 1998). En los distritos Mixe, Juchitán, Pochutla, Choapan y Tuxtepec las selvas altas

Recibido: 21 noviembre 2010; aceptado: 25 julio 2011 y medianas (Flores y Manzanero, 1999) cubrían aproximadamente el $30 \%$ de la superficie estatal. En particular el distrito de Tuxtepec, que incluye 14 municipios en el noreste de la entidad, contaba con vegetación primaria constituida por selvas medianas y altas; sin embargo, una proporción importante de la zona está siendo transformada en cultivo de caña de azúcar y áreas de pastoreo. En esta área de Oaxaca hay pocos estudios orientados a conocer la diversidad de la flora y la fauna de estos bosques tropicales, a pesar de que su pérdida es acelerada (Alfaro et al., 2006; Briones-Salas et al., 2001). Ante esta situación es fundamental conocer la biodiversidad, particularmente del grupo de los mamíferos, así como los cambios que pueden presentarse frente a estas modificaciones en el ambiente. 
Los estudios sobre la diversidad de las comunidades suelen referirse principalmente a la riqueza de especies, pero información adicional, como la de abundancia proporcional, diversidad de gremios o de algún índice de la estructura de la comunidad puede permitir una descripción más completa y detallada (Magurran, 1988). Cabe mencionar que a pesar de las desventajas que presentan, los índices se proponen como una herramienta útil para describir grupos de especies a partir de información parcial. Esta descripción de la biodiversidad, tanto de riqueza como de abundancia relativa de las especies, se incluye en un solo valor y facilita la comparación directa entre estudios en localidades diferentes (Peet, 1974; Magurran, 1988). Sirve además como indicador de cambios en la diversidad de las comunidades a través del tiempo o ante modificaciones en el ambiente (McErlean et al., 1973; Majer, 1983; Magurran, 1988).

Es importante recabar información sobre el estado de la diversidad de mamíferos que permita establecer acciones de conservación en este tipo de vegetación. Es por eso que este trabajo se enfocó a estimar la diversidad de mamíferos terrestres de talla media y grande, considerando la riqueza de especies, su abundancia y diversidad de gremios, y se complementa con índices de la estructura de la comunidad en una selva mediana del noreste de Oaxaca.

\section{Materiales y métodos}

Área de estudio. Se ubicó en la comunidad de cerro Tepezcuintle, municipio de San Miguel Soyaltepec, distrito de Tuxtepec, en el noreste del estado de Oaxaca ( $18^{\circ} 08^{\prime} 51^{\prime \prime}$ $\left.\mathrm{N}, 96^{\circ} 20^{\prime} 28^{\prime \prime} \mathrm{O}\right)$. La zona presenta una proporción alta de área dedicada a la agricultura y pastoreo $(40 \%)$, en comparación con la selva $(11 \%)$ o la vegetación secundaria $(13 \%)$. La temperatura media anual se encuentra entre los 20 y $28^{\circ} \mathrm{C}$. La precipitación media anual oscila entre 1000 y $1700 \mathrm{~mm}$. El clima predominante es de tipo (Am (i') gw", es decir, cálido húmedo con lluvias abundantes en verano (Challenger, 1998; Flores y Manzanero, 1999).

Para el registro de datos dentro de la selva mediana se establecieron 3 sendas, 2 de $3 \mathrm{~km}$ y 1 de $2 \mathrm{~km}$ de longitud. Sobre estas 3 sendas se colocó un total de 26 puntos de muestreo, separados entre si por $200 \mathrm{~m}$, cubriendo un área de 1313 ha. En cada punto se colocó un dispositivo de muestreo, procurando que 2 dispositivos similares estuvieran separados entre si por $800 \mathrm{~m}$. En total se colocaron 7 trampas Tomahawk, 7 cepos, 6 estaciones olfativas y 6 fototrampas (Cuddeback Digital Camera modelo Expert 3.0 MP). Los dispositivos fueron revisados 3 días consecutivos por mes, de septiembre de 2007 a agosto de 2008. Esta forma de recolección fue elegida por cuestiones logísticas (número de trampas disponibles y tiempo de revisión). En estudios previos se indica que para estimar algunos parámetros son suficientes los periodos de recolección cortos (Harrison et al., 2002; Sánchez et al., 2004). Asimismo, en otros estudios se sugiere que con el uso complementario de varios métodos se puede registrar un mayor número de datos para describir la diversidad (Barea-Azcón et al., 2007; Lyra-Jorge et al., 2008).

Análisis de datos. El éxito de captura se expresó como el número total de registros/esfuerzo de colecta multiplicado por $100 \mathrm{y}$ el esfuerzo de colecta como el número total de trampas colocadas por el número total de días muestreados. La riqueza de especies se tomó como el número de especies registradas en la zona por cualquier método, incluso de datos provenientes de observaciones fuera de las sendas. Para determinar si el esfuerzo de captura fue suficiente para lograr una estimación aceptable de la riqueza de especies, se evaluaron 2 modelos asintóticos de acumulación de especies, el de Clench y el de dependencia lineal, y se seleccionó el más adecuado con el criterio de máxima verosimilitud mediante el programa Species accumulation (Díaz-Francés y Soberón, 2005). Para el modelo seleccionado se calculó el valor de la asíntota, y en el caso de no alcanzarla, se calculó el esfuerzo adicional requerido para registrar el 95\% del valor asintótico predicho por el modelo seleccionado (Soberón y Llorente, 1993). Los modelos se obtuvieron a partir de una matriz de presenciaausencia de registros por especie, la cual se aleatorizó 100 veces con el programa EstimateS versión 8.0.0 (Colwell, 2000).

Se presentan valores de abundancia (número de registros para cada especie), y con fines de comparación, de abundancia relativa, expresada como la relación entre el número de registros de cada especie y el número total de registros de todas las especies. La diversidad de la comunidad se analizó mediante el índice de diversidad de Shannon-Weaver, el de equidad de Pielou y el de dominancia de Berger-Parker (Magurran, 1988). Para las estimaciones de abundancia y diversidad, sólo fueron tomados en cuenta los registros obtenidos en las trampas $\mathrm{y}$ en los recorridos en los senderos, debido a que dentro de las sendas la recolección de datos se llevó a cabo de manera estandar.

Con base en la literatura, las especies registradas se clasificaron en diferentes gremios tróficos, para conocer la diversidad de la comunidad desde este punto de vista. En este estudio se consideraron como especies de talla media y grande todas aquellas cuyos individuos adultos pesan más de 100 g (Robinson y Redford, 1986; Medellín, 1994). Para la clasificación taxonómica se siguió el sistema de Wilson y Reeder (2005). Los rastros y fotografías están depositados en la Colección de Referencia de Mamíferos, Laboratorio de Ecología Animal del Centro 
Interdisciplinario de Investigación para el Desarrollo Integral Regional, Unidad Oaxaca, Instituto Politécnico Nacional (ECOAN-MAM).

\section{Resultados}

Esfuerzo y éxito de la recolección. Se llevaron a cabo un total de 941 noches-trampa; las sendas se recorrieron en 36 ocasiones, cubriendo un total de $288 \mathrm{~km}$ durante los 12 periodos de muestreo. El éxito de captura para la zona fue de $8.61 \%$.

Diversidad. Las especies registradas representaron 6 órdenes, 11 familias y 14 géneros. El modelo asintótico de acumulación de especies de Clench fue el que mejor se ajustó a los datos, 3.65 veces mejor que el de dependencia lineal (logaritmo de la verosimilitud 241.14 vs 239.84, respectivamente). La asíntota prevista por el modelo se ubicó en 20 especies (parámetros del modelo: tasa de incremento de nuevas especies al comienzo del inventario, $\mathrm{a}=0.618878$ y parámetro relacionado con la forma de la curva, $b=0.031109$, asíntota $=a / b$ ). Para alcanzar el 95\% de la asíntota se requiere de 610 registros adicionales, dado que se registraron 15 especies.

Las especies más abundantes fueron el tejón Nasua narica con 25 registros (abundancia relativa 0.23), el pecarí de collar Pecari tajacu con 24 (0.22), el serete Dasyprocta mexicana con 16 (0.15), el tepezcuintle Cuniculus paca con 15 (0.14) y el ocelote Leopardus pardalis con $11(0.10)$. Para el resto de las especies se obtuvieron entre 1 y 8 registros ( 0.01 y 0.07 , respectivamente). No se calculó la abundancia relativa de los tlacuaches Didelphis virginiana y Philander opossum y del mapache Procyon lotor debido a que se registraron fuera de las sendas.

La diversidad expresada por medio del índice de Shannon-Weaver para la comunidad fue de 0.89; el de equitatividad, de 0.35 y el de dominancia de Berger-Parker, de 0.23 , con el tejón como la especie numéricamente predominante. Estuvieron representados 5 gremios alimenticios dentro de la comunidad: el omnívoro con 6 especies, el frugívoro-herbívoro y el carnívoro con 3 cada uno, el insectívoro con 2 y el herbívoro con 1 especie (Cuadro 1).

\section{Discusión}

En este estudio se documentó que la comunidad de mamíferos terrestres no voladores de talla media y grande de la selva mediana de cerro Tepezcuintle está constituida por 15 especies. Entre las que se registran por primera vez para la zona están N. narica, tejón; Canis latrans, coyote; Urocyon cinereoargenteus, zorra gris; Leopardus wiedii, tigrillo; Eira barbara, viejo de monte; Dasypus novemcinctus, y armadillo de nueve bandas y P. tajacu, pecarí de collar. Por el contrario, 4 especies que se han registrado en otros estudios no fueron detectadas en éste: Didelphis marsupialis, tlacuache, Puma yagouaroundi, jaguarundi, Sphiggurus mexicanus, puerco espín y Mazama americana, venado temazate.

Al considerar las 15 especies registradas aquí, más las otras 4 cuya presencia se había documentado previamente en la zona, la composición confirmada de la fauna de mamíferos silvestres en el distrito de Tuxtepec es de 19 (Cuadro 1), mientras que el modelo de acumulación de especies de Clench indica que el valor asintótico es de 20, es decir, en la zona de cerro Tepezcuintle falta por registrar al menos una especie más.

En otras selvas altas y medianas del estado de Oaxaca se han registrado entre 10 y 28 especies de mamíferos de talla media y grande. Los Chimalapas es hasta ahora la zona con mayor riqueza de especies (Alfaro Espinosa et al., 2006; Lira Torres, 2006; Olguín-Monroy et al., 2008), mientras que en selvas de Chiapas y Yucatán se han encontrado entre 10 y 31 (March y Aranda, 1992; Cruz-Lara et al., 2004; Faller-Menéndez et al., 2005). El mayor número (31), se encontró en la Selva Lacandona, Chiapas (March y Aranda, 1992).

Pecari tajacu, D. mexicana, D. novemcinctus, $N$. narica y C. paca fueron las especies más abundantes en este estudio, lo que coincide con lo observado en otras selvas, tanto de Oaxaca (Alfaro Espinosa et al., 2006) y de Chiapas (March y Aranda, 1992), como de la Amazonia ecuatoriana (Zapata-Ríos et al., 2002), la isla Barro Colorado, Panamá (Eisenberg y Thorington, 1973) y el Parque Nacional Madidi, Bolivia (Gómez et al., 2001). Estas especies predominan en diferentes regiones de México, Centro y Sudamérica, probablemente por su adaptabilidad a ambientes perturbados o a la plasticidad de sus hábitos alimenticios. En el primer caso se encuentran N. narica y D. novemcintus (Gompper, 1995; Mendoza-Durán, 2005); en el segundo, P. tajacu, D. mexicana y C. paca; por ejemplo, la dieta de C. paca está constituida principalmente de frutos, que consume de acuerdo a su disponibilidad en el ambiente a lo largo del año (Pérez, 1992).

Por su parte, C. latrans y $U$. cinereoargenteus se encontraron con abundancias bajas en la zona de estudio, a pesar de que se les considera especies comunes (Servín y Chacón, 2005, 2005a). En este estudio se encontraron rastros de $P$. opossum y de $P$. lotor en sitios cercanos a los cultivos y en las orillas de los arroyos aledaños, como se ha observado en otros sitios (Castro-Arellano y Medellín, 2005; Valenzuela-Galván, 2005). Aunque estas especies se consideran generalistas y adaptables a una considerable diversidad de ambientes, no se registraron en el interior del bosque tropical, $\mathrm{y}$ al parecer prefieren zonas cercanas a cultivos o cuerpos de agua. 
Cuadro 1. Mamíferos terrestres de talla media y grande de la comunidad de Cerro Tepezcuintle, Tuxtepec, Oaxaca

\begin{tabular}{|c|c|c|c|c|}
\hline Taxa & Gremio trófico & Número de registros & Abundancia relativa & Estado de conservación \\
\hline \multicolumn{5}{|l|}{ Orden Didelphimorphia } \\
\hline \multicolumn{5}{|l|}{ Familia Didelphidae } \\
\hline Didelphis virginiana ${ }^{\mathrm{b}, \mathrm{d}}$ & $\mathrm{O}$ & 9 & -- & \\
\hline Didelphis marsupialis $\mathrm{s}^{\mathrm{a}, \mathrm{b}, \mathrm{c}}$ & $\mathrm{O}$ & -- & -- & \\
\hline Philander opossum ${ }^{\mathrm{a}, \mathrm{b}, \mathrm{c}, \mathrm{d}}$ & $\mathrm{O}$ & 1 & -- & \\
\hline \multicolumn{5}{|l|}{ Orden Xenarthra } \\
\hline \multicolumn{5}{|l|}{ Familia Dasypodidae } \\
\hline Dasypus novemcinctus ${ }^{\mathrm{d}}$ & I & 8 & 0.07 & \\
\hline \multicolumn{5}{|l|}{ Familia Myrmecophagidae } \\
\hline Tamandua mexicana $a^{\mathrm{b}, \mathrm{d}}$ & I & 1 & 0.01 & $\mathrm{P}$ \\
\hline \multicolumn{5}{|l|}{ Orden Carnivora } \\
\hline \multicolumn{5}{|l|}{ Familia Canidae } \\
\hline Canis latrans ${ }^{\mathrm{d}}$ & $\mathrm{C}$ & 2 & 0.02 & \\
\hline Urocyon cinereoargenteus $\mathrm{d}^{\mathrm{d}}$ & $\mathrm{O}$ & 1 & 0.01 & \\
\hline \multicolumn{5}{|l|}{ Familia Felidae } \\
\hline Leopardus pardalis ${ }^{\mathrm{d}}$ & $\mathrm{C}$ & 11 & 0.10 & $\mathrm{P}$ \\
\hline Leopardus wiedii ${ }^{\mathrm{d}}$ & $\mathrm{C}$ & 2 & 0.02 & $\mathrm{P}$ \\
\hline Puma yagouaround $i^{\mathrm{a}, \mathrm{b}, \mathrm{c}}$ & $\mathrm{C}$ & -- & -- & A \\
\hline \multicolumn{5}{|l|}{ Familia Mustelidae } \\
\hline Eira barbara ${ }^{\mathrm{d}}$ & $\mathrm{O}$ & 2 & 0.02 & $\mathrm{P}$ \\
\hline \multicolumn{5}{|l|}{ Familia Procyonidae } \\
\hline Nasua narica ${ }^{\mathrm{d}}$ & $\mathrm{O}$ & 25 & 0.23 & \\
\hline Procyon lotor ${ }^{\mathrm{b}, \mathrm{d}}$ & $\mathrm{O}$ & 1 & -- & \\
\hline \multicolumn{5}{|l|}{ Orden Artiodactyla } \\
\hline \multicolumn{5}{|l|}{ Familia Tayassuidae } \\
\hline Pecari tajacu ${ }^{\mathrm{d}}$ & $\mathrm{F}, \mathrm{H}$ & 24 & 0.22 & \\
\hline \multicolumn{5}{|l|}{ Familia Cervidae } \\
\hline Mazama american $a^{\mathrm{a}, \mathrm{b}, \mathrm{c}}$ & $\mathrm{F}, \mathrm{H}$ & -- & -- & \\
\hline \multicolumn{5}{|l|}{ Orden Rodentia } \\
\hline \multicolumn{5}{|l|}{ Familia Erethizontidae } \\
\hline Sphiggurus mexicanus ${ }^{\mathrm{b}}$ & $\mathrm{H}$ & -- & -- & A \\
\hline \multicolumn{5}{|l|}{ Familia Cuniculidae } \\
\hline Cuniculus paca ${ }^{\mathrm{b}, \mathrm{d}}$ & $\mathrm{F}$ & 15 & 0.14 & \\
\hline \multicolumn{5}{|l|}{ Familia Dasyproctidae } \\
\hline Dasyprocta mexicanab, & $\mathrm{F}$ & 16 & 0.15 & \\
\hline \multicolumn{5}{|l|}{ Orden Lagomorpha } \\
\hline \multicolumn{5}{|l|}{ Familia Leporidae } \\
\hline Sylvilagus brasiliensis ${ }^{\mathrm{b}, \mathrm{d}}$ & $\mathrm{H}$ & 1 & 0.01 & \\
\hline Total & & 119 & 1.000 & \\
\hline
\end{tabular}

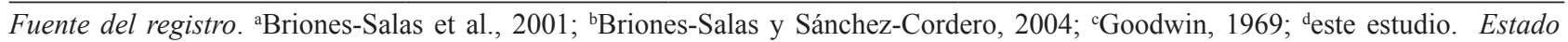
de conservación (NOM-059-ECOL-2000). A, amenazada; P, en peligro de extinción. Gremio trófico. C, carnívoro; F, frugívoro; H, herbívoro; I, insectívoro; O, omnívoro.

El oso hormiguero, Tamandua mexicana, L. wiedii y E. barbara, se registraron con abundancias bajas, lo que parece común en sitios tropicales en Sudamérica, como Los Llanos, Venezuela (Sunquist et al., 1989), Panamá (Glenz, 1997), Belice y Bolivia (Konecny, 1989; Cuellar et al., 2006).

La diversidad de cerro Tepezcuintle expresada por medio del índice de Shannon-Weiner (0.89) fue baja comparada con la obtenida en otros lugares del estado de
Oaxaca, como la Chinantla baja (1.05, Alfaro Espinosa et al., 2006) o en la Selva Lacandona, Chiapas (2.33, Cruz-Lara et al., 2004). A pesar de esto, el valor de cerro Tepezcuintle radica en la presencia de L. pardalis, L. wiedii, E. barbara y T. mexicana, especies que se encuentran en riesgo según la NOM-059 (SEMARNAT, 2002). Por otra parte, la presencia de T. tajacu, D. mexicana y C. paca es relevante porque son especies de importancia para los pobladores locales, ya que son preferidas por los cazadores 
y son presas de mamíferos carnívoros medianos y grandes. En la comunidad se registró a $L$. wiedii, E. barbara y $T$. mexicana, especies que están asociadas a la presencia de las selvas, dado sus hábitos arborícolas (Konecny, 1989; Sunquist et al., 1989), así como D. mexicana, una especie de distribución restringida, asociada a selvas (Arita, 2005), cuya presencia podría estar en riesgo ante la pérdida de este ambiente en la zona.

\section{Agradecimientos}

A las autoridades comunales de cerro Tepezcuintle por la autorización para realizar el estudio. El CONACYT proporciona una beca de estudios de posgrado para el primer autor, y el Instituto Politécnico Nacional el financiamiento para la ejecución del proyecto (apoyos SIP-20070826 y SIP-20080431 al segundo autor). A. Alfaro E., J. L. García G., S. García O., S. Guevara A., A. E. Hernández C., E. E. Pérez, C., D. G. Rodríguez C., A. Santiago M. y Y. Zacarías E. apoyaron en el trabajo de campo a lo largo de este estudio.

\section{Literatura citada}

Alfaro-Espinosa, A. M., J. L. García-García y A. Santos-Moreno. 2006. Mamíferos de los municipios Santiago Jocotepec y Ayotzintepec, Chinantla Baja, Oaxaca. Naturaleza y Desarrollo 4:19-23.

Arita, H. T. 2005. Dasyprocta mexicana. In Los mamíferos silvestres de México, G. Ceballos y G. Oliva (coords.) Comisión Nacional para el Conocimiento y Uso de la Biodiversidad/ Fondo de Cultura Económica, México, D.F. p. 817-818.

Barea-Azcón, J. M., E. Virgós, E. Ballesteros-Duperón, M. Moleón y M. Chirosa. 2007. Surveying carnivores at large spatial scales: a comparison of four broad-applied methods. Biodiversity and Conservation 16:1213-1230.

Briones-Salas, M. A., V. Sánchez-Cordero y A. G. Quintero. 2001. Lista de mamíferos terrestres del norte de Oaxaca. Anales del Instituto de Biología, Serie Zoología 72:125-161.

Briones-Salas, M. A. y V. Sánchez-Cordero. 2004. Mamíferos. In Biodiversidad de Oaxaca, A. J. García-Mendoza, M. J. Ordóñez y M. A. Briones (eds.) Instituto de Biología, Universidad Nacional Autónoma de México/ Fondo Oaxaqueño para la Conservación de la Naturaleza/ Word Wildlife Fund, México, D.F. p. 423-447.

Castro-Arellano, I. y R. A. Medellín. 2005. Philander opossum. In Los mamíferos silvestres de México, G. Ceballos y G. Oliva (coords.). Comisión Nacional para el Conocimiento y Uso de la Biodiversidad/ Fondo de Cultura Económica, México, D.F. p. 111-113.

Challenger, A. 1998. Utilización y conservación de los ecosistemas terrestres de México. Diversidad, presente y futuro. Fundación Sierra Madre/ Instituto de Biología, UNAM/ Comisión Nacional para el Conocimiento y Uso de la Biodiversidad, México, D. F.

Colwell, R. K. 2000. EstimateS version 6.0b1. Department of Ecology and Evolutionary Biology, University of Connecticut, Storrs.

Cruz-Lara, L. E., C. Lorenzo, L. Soto, E. Naranjo y N. RamírezMarcial. 2004. Diversidad de mamíferos en cafetales y selva mediana de las cañadas de la Selva Lacandona, Chiapas, México. Acta Zoológica Mexicana (nueva serie) 20:63-81.

Cuellar, E., L. Maffei, R. Arispe y A. Noss. 2006. Greffroy's cats at the northern limit of their range: activity patterns and density estimates from camera trapping in Bolivian dry forests. Studies on Neotropical Fauna and Environment 41:169-177.

Díaz-Francés, E. y J. Soberón. 2005. Statistical estimation and model selection of species-accumulation functions. Conservation Biology 19:569-573.

Eisenberg, J. F. y R. W. Thorington, Jr. 1973. A preliminary analysis of a Neotropical mammal fauna. Biotropica 5:150161.

Faller-Menéndez, J. C., T. Urquiza-Haas, C. Chávez, S. Johnson y G. Ceballos. 2005. Registros de mamíferos en la reserva privada El Zapotal, en el noreste de la península de Yucatán. Revista Mexicana de Mastozoología 9:128-140.

Flores, A. y G. Manzanero. 1999. Los tipos de vegetación del estado de Oaxaca. In Vegetación y flora. M. A. VásquezDávila (ed.). Serie Sociedad y Naturaleza en Oaxaca 3, Instituto Tecnológico Agropecuario de Oaxaca/ Carteles, Oaxaca, Oaxaca. p. 7-45.

Glenz, W. E. 1997. Densidades de población de mamíferos en sitios protegidos y de caza en el Panamá central. In Uso y conservación de la vida silvestre neotropical, J. G. Robinson, K. H. Redford y J. E. Rabinovich (comps). Fondo de Cultura Económica, México, D.F. p. 201-213.

Goodwin, G. G. 1969. Mammals from the State of Oaxaca, Mexico, in the American Museum of Natural History. Bulletin of the American Museum of Natural History 141:1270.

Gómez, H., R. B. Wallace y C. Veitch. 2001. Diversidad y abundancia de mamíferos medianos y grandes en el noreste del área de influencia del Parque Nacional Madidi durante la época húmeda. Ecología en Bolivia 36:17-29.

Gompper, M. E. 1995. Nasua narica. Mammalian Species 487:110.

Harrison, R., D. J. Barr y J. W. Dragoo. 2002. A comparison of population survey techniques for swift foxes (Vulpes velox) in New Mexico. American Midland Naturalist 148:320-337.

Konecny, M. J. 1989. Movement patterns and food habits of four sympatric carnivore species in Belize, Central America. In Advances in Neotropical Mammalogy, K. H. Redford y J. 
F. Eisenberg (eds.) Sandhill Crane. Gainesville, Florida. p. 243-264.

Lira-Torres, I. 2006. Abundancia, densidad, preferencia de hábitat y uso de los vertebrados en la Tuza de Monroy, Santiago Jamiltepec, Oaxaca. Revista Mexicana de Mastozoología 10:41-66.

Lyra-Jorge, M. C., G. Ciochete, V. R. Pivello y S. T. Meirelles. 2008. Comparing methods for sampling large- and mediumsized mammals: camera trap and track plots. European Journal of Wildlife Researches 54:739-744.

Magurran, A. E. 1988. Ecological diversity and its measurement. Chapman and Hall, London. 179 p.

Majer, J. D. 1983. Ants: bio-indicator of minesite rehabilitation, land-use, and land conservation. Environmental Management 7:375-383.

March, I. J. y M. Aranda. 1992. Mamíferos de la selva lacandona, Chiapas. In Reserva de la Biosfera Montes Azules, Selva Lacandona: Investigaciones para su conservación. M. A. Vázquez-Sánchez y M. A. Ramos-Olmos (comps.) Publicaciones Especiales Ecosfera 1. Centro de Estudios para la Conservación de los Recursos Naturales, México, D.F. p. 201-220.

McErlean, A. J., S. G. O'Connor, J. A. Mihursky y C. I. Gibson. 1973. Abundance, diversity and seasonal patterns of estuarine fish populations. Estuarine and Coastal Marine Science 1:1936.

Medellín, R. A. 1994. Mammal diversity and conservation in the Selva Lacandona, Chiapas, Mexico. Conservation Biology 8:780-799.

Mendoza-Durán, A. 2005. Dasypus novemcinctus. In Los mamíferos silvestres de México, G. Ceballos y G. Oliva (coords.). Comisión Nacional para el Conocimiento y Uso de la Biodiversidad/ Fondo de Cultura Económica, México, D.F. p. $117-118$

Olguín-Monroy, H., L. León Paniagua, U. M. Samper-Palacios y V. Sánchez-Cordero Dávila. 2008. Mastofauna de la región de los Chimalapas, Oaxaca, México. In Avances en el estudio de los mamíferos de México, vol. II. E. Espinoza-Medinilla, C. Lorenzo-Monterrubio y J. Ortega (eds.) Publicaciones especiales. Asociación Mexicana de Mastozoología, México, D. F. p. $165-216$.

Peet, R. K. 1974. The measurement of species diversity. Annual Reviews Ecology and Systematic 5:285-307.

Pérez, E. M. 1992. Agouti paca. Mammalian Species 404:1-7.
Robinson, J. G. y K. H. Redford. 1986. Body size, diet, and population density of Neotropical forest mammals. The American Naturalist 128:665-680.

Sánchez, F., P. Sánchez-Palomino y A. Cadena. 2004. Inventario de mamíferos en un bosque de los Andes centrales de Colombia. Caldasia 26:291-309.

SEMARNAT (Secretaría del Medio Ambiente y Recursos Naturales). 2002. Norma Oficial Mexicana NOM-059ECOL-2001. Protección ambiental, especies de flora y fauna silvestre de México, categorías de riesgo y especificaciones para su inclusión, exclusión o cambio, y lista de especies en riesgo. Diario Oficial de la Federación, 6 de marzo.

Servin, J. y E. Chacón. 2005. Canis latrans. In Los mamíferos silvestres de México, G. Ceballos y G. Oliva (coords.) Comisión Nacional para el Conocimiento y Uso de la Biodiversidad/ Fondo de Cultura Económica, México, D.F. p.349-350

Servin, J. y E. Chacón. 2005a. Urocyon cinereoargenteus. In Los mamíferos silvestres de México, G. Ceballos y G. Oliva (coords.). Comisión Nacional para el Conocimiento y Uso de la Biodiversidad/ Fondo de Cultura Económica, México, D.F. p. 354-355.

Soberón, J. y J. B. Llorente. 1993. The use of species accumulation functions for the prediction of species richness. Conservation Biology 7:480-488.

Sunquist, M. E., F. Sunquist y D. E. Danake. 1989. Ecological separation in a Venezuelan Llanos carnivore community. In Advances in Neotropical Mammalogy, K. H. Redford y J. F. Eisenberg (eds.) Sandhill Crane, Gainesville, Florida. p. 197-232

Valenzuela-Galván, D. 2005. Procyon lotor. In Los mamíferos silvestres de México, G. Ceballos y G. Oliva (coords.). Comisión Nacional para el Conocimiento y Uso de la Biodiversidad/ Fondo de Cultura Económica, México, D.F.. p. 415-417.

Wilson, D. E. y D. M. Reeder (eds.). 2005. Mammals species of the world, a taxonomic and geographic reference, tercera edición. Johns Hopkins University Press, Baltimore, Maryland. 2142 p.

Zapata-Ríos, G., E. Araguillin y J. P. Jorgenson. 2002. Caracterización de la comunidad de mamíferos no voladores en las estribaciones orientales de la cordillera del Kutukú, Amazonia ecuatoriana. Mastozoología Neotropical 13:227238 . 